\title{
EXISTENCE THEOREMS FOR SOLUTIONS TO SET-VALUED STOCHASTIC DIFFERENTIAL EQUATIONS AND APPLICATIONS
}

\author{
$\mathrm{Ho} \mathrm{Vu}{ }^{1}$, Ngo Van $\mathrm{Hoa}^{2}$ \\ ${ }^{1}$ Faculty of Accounting-Auditing,University of Hung Vuong, Ho Chi Minh City, Vietnam \\ ${ }^{2}$ Faculty of Mathematics and Computer Science, University of Science, VNU Ho Chi Minh City, \\ Vietnam
}

Keywords: Set-Valued Stochastic Differential Equations, Existence Theorems, Hukuhara derivative, interval-valued stochastic.

\begin{abstract}
In this paper, a class of new stochastic differential equations on semilinear Hausdorff space under Hukuhara derivative, called set-valued stochastic differential equations (SSDEs) driven by a Wiener process. Moreover, some corresponding properties of SSDEs are discussed such as existence, uniqueness of solution. Finaly, we give some applications to models of interval-valued stochastic differential equations such as stock prices model and the Langevin equation.
\end{abstract}

\section{INTRODUCTION}

The set-valued differential and integral equations are important parts of the set-valued analysis theory and they have the important value of theory and application in control theory, and this was started in 1969 by De Blasi and Iervolino [1]. Therefore, set-valued differential equations have been studied by many authors due to many application. Reader can refer to the very good book and papers (see $[6,16,5,10,11,13,9,15]$ and references therein). Beside that, a new class of set-valued differential equations on semilinear Hausdorff space under classic Hukuhara derivative, called setvalued stochastic differential equations (SSDEs) which as far as we known, that the problem of the properties of solutions to set-valued stochastic differential equations is still open.

In classical real analysis, maybe one of the most important concepts is that of the derivative of a real-valued function. Correspondingly, in set-valued analysis or in the theory of differential inclusions, we would expect to have a notion of the derivative of a set-valued. Instead, the classical derivatives are used in both research directions which we have mentioned above. The reason for this is that a derivative concept which is both theoretically well founded and it is also applicable to concrete situations is still missing, despite the almost half a century of (otherwise very important) development of these domains.

Therefore, this was started in 1967 by Hukuhara who given Hukuhara derivative of a set-valued mapping and it has been studied in several works. The paper of Hukuhara was the starting point for the topic of set differential equations (see $[6,5,10,11,13,9,15]$ and references therein ) and later also for fuzzy differential equations. Also, as a very important generalization and development related to the subject of the present paper is in the field of fuzzy sets, i.e., fuzzy calculus and fuzzy differential equations. Recently, several works as e.g., $[4,17,7,12,3,2,8,14]$, have brought back into the attention of the nonlinear analysis community. Beside that, recently, some studies about stochastic differential inclusion (SDIs)(see e.g. [19,20,21] and references therein), stochastic fuzzy differential equations (SFDEs), (see e.g. [29,30] and references therein) and stochastic set differential equations (SSDEs)(see e.g. [22,25,26,27,28] and references therein). Indeed, it is well know that the classical type stochastic differential equation is

$$
\left\{\begin{array}{l}
d x_{t}=f\left(t, x_{t}\right) d t+g\left(t, x_{t}\right) d w_{t} \\
x(0)=x_{0}
\end{array}\right.
$$


or the stochastic integral form $x_{t}=\eta+\int_{0}^{t} f\left(s, x_{s}\right) d t+\int_{0}^{t} g\left(s, x_{s}\right) d w_{s}$ where $w_{t}$ is m-dimiential

Winer process $f:[0, T] x R^{n} \rightarrow R^{n}, g:[0, T] \mathrm{x} R^{n} \rightarrow R^{n x m}$ are Borel measurable functions with some conditions. It describle movement law of an object with random disturbing given the initial condition. In complex systems, however we can not decide exactly $x_{t}$ at time $t$ but we can known it take valued for instance, in a interval $X_{t}=\left[x_{t}^{1}, x_{t}^{2}\right]$, where $x_{t}^{1}<x_{t}^{2}$

This leads us to a set-valued stochastic differential equations as follows

$$
\left\{\begin{array}{l}
X^{\prime}{ }_{t}=F\left(t, X_{t}\right)+G\left(t, X_{t}\right) \xi(t) \\
X(0)=X_{0}
\end{array}\right.
$$

where $F:[0, T] \times K_{C C}\left(R^{n}\right) \rightarrow K_{C C}\left(R^{n}\right), G:[0, T] \times K_{C C}\left(R^{n}\right) \rightarrow K_{C C}\left(R^{n}\right)$. We note that $F, G$ take set-valued in $K_{C C}\left(R^{n}\right)$. Let us consider an example, of a simple population growth model $d x_{t}=c_{t} x_{t} d t, x_{0}=c$, where $x_{t}$ is the size of the population at time $t$, and $c_{t}$ is the relative rate of growth at time $t$. In fact, $c_{t}$ might happen that it is not completely known, but subject to some random environmental effects. Hence we have $c_{t}=a_{t}+$ "noise", where $a_{t}$ is a non-random function, so that (4) can be written as $d x_{t}=a_{t} x_{t} d t+h_{t} x_{t} d w_{t}, x=c$. By the complexity of the real world, we cannot know the exact size of the population at time $t$, but we can estimate the interval it takes in or it uctuates within. In other words, $x_{t} \in X_{t}=\left[X_{t}^{L}, X_{t}^{U}\right]$. This leads us to interval-valued stochastic differential equation $X^{\prime}{ }_{t}=c_{t} X_{t}+h_{t} X_{t} \xi(t)$. Where ${ }^{\xi(t)}$ one-dimensional "white noise", with "white noise" is the time derivative of one-dimensional Wiener process, i.e., $\frac{d w_{t}}{d t}=\xi(t)$ In general, a setvalued stochastic differential equation is given as (1.1).

We organize this paper as follows: In section 2, we recall some basic concepts and notations which are useful in next sections. In section 3 we introduce set-valued stochastic process and set-valued stochastic integral with respect to 1-dimensional Brownian motion, and discuss some of its properties. In section 4, we present the existence, uniqueness properties of set-valued solutions to SSDEs. In section 5, we give the examples and further research of this paper.

\section{PRELIMINARIES}

We recall some notations and concepts presented in detail in recent series works of $\mathrm{V}$. Lakshmikantham et al (see [6]). Let $K_{C C}\left(R^{n}\right)$ denote the collection of all nonempty compact convex subsets of $R^{n}$. Given $A, B \in K_{C C}\left(R^{n}\right)$ the Hausdorff distance between $\mathrm{A}$ and $\mathrm{B}$ is defined by

$$
d_{H}(A, B)=\max \{\underset{a \in A, b \in B}{\operatorname{supinf}}\|a-b\| ; \underset{b \in B, a \in A}{\operatorname{supinf}}\|b-a\|\}
$$

And $\left\{\theta^{\mathrm{n}}\right\}$-the zero points set in $K_{C C}\left(R^{n}\right)$. It is known that $\left(K_{C C}\left(R^{n}\right), d_{H}\right)$ is a complete metric space and $K_{C C}\left(R^{n}\right)$ is a complete and separable with respect to $d_{H}$.

We define the magnitude of a nonempty subset $\mathrm{A}$ as,

$$
d_{H}\left(A, \theta^{n}\right)=\square A \square=\sup \{\square a \square, a \in A\}
$$


The Hausdorff metric satisfes the properties below :

i) $d_{H}(A+C, B+C)=d_{H}(A, B)$ and $d_{H}(A, B)=d_{H}(B, A)$,

ii) $d_{H}(\lambda A, \lambda B)=\lambda d_{H}(A, B)$,

iii) $d_{H}(A, B) \leq d_{H}(A, C)+d_{H}(C, B)$,

iv) $d_{H}\left(A+A^{\prime}, B+B^{\prime}\right) \leq d_{H}(A, B)+d_{H}\left(A^{\prime}, B^{\prime}\right)$

for all $A, B, C, A^{\prime}, B^{\prime} \in K_{C C}\left(R^{n}\right)$ and $\lambda \in R_{+}$. If $\alpha, \beta \in R$ and $A, B \in K_{C C}\left(R^{n}\right)$, then

$$
\alpha(A+B)=\alpha A+\alpha B, \quad \alpha(\beta A)=(\alpha \beta) A, \quad 1 . A=A
$$

Let $A, B \in K_{C C}\left(R^{n}\right)$. The set $C \in K_{C C}\left(R^{n}\right)$ satisfying $A=B+C$ is called the Hausdorff difference (the geometric difference) of the sets $A$ and $B$ and is denoted by $A-B$. Given an interval $I$ in $R_{+}$, we say that the set-valued stochastic mapping $X: I \times \Omega \rightarrow K_{C C}\left(R^{n}\right)$ has a Hukuhara derivative $X^{\prime}\left(t_{0}, \cdot\right)$ at a point $t_{0} \in I$, if

$$
\lim _{h \rightarrow 0^{+}} \frac{X\left(t_{0}+h, \cdot\right)-X\left(t_{0}, \cdot\right)}{h} \text { and } \lim _{h \rightarrow 0^{+}} \frac{X\left(t_{0}, \cdot\right)-X\left(t_{0}-h, \cdot\right)}{h}
$$

exist in the topology of $K_{C C}\left(R^{n}\right)$ and are equal, to what we then call $X^{\prime}\left(t_{0},{ }^{-}\right)$.

By embedding $K_{C C}\left(R^{n}\right)$ as a complete cone in a corresponding Banach space and taking into account results on the differentiation of Bochner integral, we find that if

$$
X(t, \cdot)=X_{0}+\int_{t_{0}}^{t} \Phi(s, \cdot) d s, \quad X_{0}: \Omega \rightarrow K_{C C}\left(R^{n}\right),
$$

Where $\Phi: I \times \Omega \rightarrow K_{c}\left(R^{n}\right)$ is integrable in the sense of Bochner, then $X^{\prime}(t, \cdot)$ exists and the equality $X^{\prime}(t, \cdot)=\Phi(t, \cdot)$ a.e on $I \times \Omega$ holds.

The Hukuhara integral of $\mathrm{X}$ is given by

$$
\int_{t} X(s, \cdot) d s=\operatorname{cl}\left[\int_{t} x(s, \cdot) d s: x \text { is a continuous selector of } X\right]
$$

for any compact set $I \subset R_{+}$

Some properties of the Hukuhara integral below :

If $X: I \times \Omega \rightarrow K_{c c}\left(R^{n}\right)$ is integrable, one has

$$
\int_{t_{0}}^{t_{2}} X(s, \cdot) d s=\int_{t_{0}}^{t_{1}} X(s, \cdot) d s+\int_{t_{1}}^{t_{2}} X(s, \cdot) d s, \quad t_{0} \leq t_{1} \leq t_{2}
$$

and

$$
\int_{t_{0}}^{t} \lambda X(s, \cdot) d s=\lambda \int_{t_{0}}^{t} X(s, \cdot) d s, \quad \lambda \in R
$$

If $X_{1}, X_{2}: I \times \Omega \rightarrow K_{C C}\left(R^{n}\right)$ are integrable, then $d_{H}\left(X_{1}(\cdot, \cdot), X_{2}(\cdot, \cdot)\right): I \times \Omega \rightarrow R$ is integrable and $d_{H}\left(\int_{t_{0}}^{t} X_{1}(s, \cdot) d s, \int_{t_{0}}^{t} X_{2}(s, \cdot) d s\right) \leq \int_{t_{0}}^{t} d_{H}\left(X_{1}(s, \cdot), X_{2}(s, \cdot)\right) d s$.

\section{SET-VALUED STOCHASTIC PROCESS AND SET-VALUED STOCHASTIC INTEGRALS}

Let $(\Omega, \mathrm{F}, \mathrm{P})$ a complete filtered probability space with a filtration $\left\{_{t}\right\}_{t \in[0, I]}$ satisfying the usual conditions, i.e $\left\{F_{t}\right\}_{t \geq 0}$ is increasing and right continuous family of $\sigma$-subalgebras of $F$ and $F_{0}$ contains all $P$ - null sets. Let $w_{t}, t \in[0, T]$ be an $F_{t}$-adapted one-dimensional Wiener process 
defined on $(\Omega, F, P)$. We shall deal with measurable multifunctions defined on $\Omega$ with values in the family of nonempty closed subsets of $K_{C C}\left(R^{n}\right)$. Let $X \in L^{2}\left(\Omega, F ; K_{C C}\left(R^{n}\right)\right)$ we define $S^{2}(X)=\left\{x \in L^{2}\left(\Omega, F ; R^{n}\right): x(\omega) \in X(\Omega)\right.$ a.e. $\left.\omega \in \Omega\right\}$. Here $L^{2}\left(\Omega, F ; R^{n}\right)$ is the space of all vectorvalued random variables $x$ such that $\|x\|_{2}^{2}=E\left[|x|^{2}\right]<\infty$ where $E[x]$ is expectation of a random variable $x$.

In this section, we call $\left(X_{t}\right)_{t \geq 0}$ a set-valued stochastic process with valued in $K_{C C}\left(R^{n}\right)$ or, for short a set-valued $K_{C C}\left(R^{n}\right)$-process if $X:[0, \infty) \times \Omega \rightarrow K_{C C}\left(R^{n}\right)$ is a set-valued function such that

i) For any $t \in[0, \infty), X_{t}=X(t, \omega)$ is closed set convex in $K_{C C}\left(R^{n}\right)$ a.a. $\omega \in \Omega$

ii) For any fixed $t \in[0, \infty), X(t,)^{\prime}$ is a random set, that is, for Borel sets $A \in B\left(R^{n}\right)$ iii)

$$
\{\omega \in \Omega: X(t, \omega) \cap A \neq \varnothing\} \in F \text {. }
$$

A set-valued $K_{C C}\left(R^{n}\right)$-process $\left(X_{t}\right)_{t \geq 0}$ is called $F_{t}$-adapted if $X_{t}$ is measurable with respect to $F_{t}$ for every $t \geq 0$, and a set-valued $K_{C C}\left(R^{n}\right)$-process $\left(X_{t}\right)_{t \geq 0}$ is called measurable if $\{(t, \omega) \in[0, \infty) \times \Omega: X(t, \omega) \cap A \neq \varnothing\} \in B([0, \infty) \times F)$ for $A \in B\left(R^{n}\right)$

In [19], authors showed that for any $X \in L^{2}\left(\Omega, F ; K_{C C}\left(R^{n}\right)\right.$ and sub- $\sigma$ - field $B \subset F$, there exists a unique $Y \in L^{2}\left(\Omega, B, P ; K_{C C}\left(R^{n}\right)\right)$ such that $S^{2}(Y)=\operatorname{cl}\left\{E[x|B|]: x \in S^{2}(X)\right\}$. Where the closure is taken with respect to norm in $L^{2}\left(\Omega, B ; R^{n}\right)$. This random set $Y$ is called the set-valued conditional expectation of $X$ given $B$ and we denote it by $E[X|B|]$.

Definition 3.1. A set-valued $K_{C C}\left(R^{n}\right)$-process $\left(X_{t}\right)_{t \geq 0}$ is called an $F_{t}$-martingale if

i) For any $t \geq 0, X_{t}$ is $\mathrm{L}^{1}$ - bounded;

ii) $\left(X_{t}\right)_{t \geq 0}$ is $F_{t}$-adapted;

iii) for $t \geq s \geq 0, E\left[X_{t}\left|F_{s}\right|\right]=X_{s}$ a.s.

For any random set $X \in L^{1}\left(\Omega, F ; K_{C C}\left(R^{n}\right)\right), X_{t}=E\left[X\left|F_{t}\right|\right]$ defines a set-valued $F_{t}$ martingale. The following theorem examines an important example of a set-valued martingale.

Theorem 3.1. ([22]) Assume that ${ }^{(m(t))_{t \geq 0}}$ is a nonnegative martingale in $L^{2}(R)$. Let $M=[-1,1]$ and define $(M(t))_{t \geq 0}$ by $M(t)=m(t) M$ a.s. Then $(M(t))_{t \geq 0}$ is a set-valued $F_{t}$ - martingale. For a set-valued $K_{C C}\left(R^{n}\right)$-process $\left(X_{t}\right)_{t \geq 0}$, an $L^{2}$-selection of $\left(X_{t}\right)_{t \geq 0}$ is a real valued process $(x(t))_{t \geq 0} \in L^{2}\left(R^{n}\right) \quad$ satisfying for every $t \geq 0, x(t, \omega) \in X(t, \omega)$ a.a. $\omega \in \Omega$. We denote by $S_{2}\left(X_{t}\right)$ the set of all $L^{2}$-selection of the set-valued $K_{C C}\left(R^{n}\right)$ - process $\left(X_{t}\right) t \geq 0$. Distinguish $S_{2}\left(X_{t}\right)$ from the set $S^{2}\left(X_{t}\right)$ of all selection $y \in L^{2}\left(\Omega, F_{t} ; R^{n}\right)$ of a random set $X_{t}$ for any fixed $t \geq 0$.

Definition 3.2. A set-valued $K_{C C}\left(R^{n}\right)$ - process $\left(X_{t}\right)_{t \geq 0}$ is called $L^{2-}$ bounded if there is a process $(h(t))_{t \geq 0} \in L^{2}(R)$ such that $\|x\|_{2} \leq h(t, \omega)$ for any $t \geq 0, \omega \in \Omega$. and $x \in X(t, \omega)$ 
Lemma 3.1. A set-valued $K_{C C}\left(R^{n}\right)$-process $\left(X_{t}\right)_{t \geq 0}$ is called $L^{2-}$ bounded if the set-valued mapping $(t, \omega): \mapsto d_{H}\left(X, \theta^{n}\right)$ is in $L^{2}\left(\Omega, F_{t} ; K_{C C}\left(R^{n}\right)\right)$.

Let $L^{2}\left(\Omega, F_{t} ; K_{C C}\left(R^{n}\right)\right)$ be the set of all $K_{C C}\left(R^{n}\right)$ valued $L^{2-}$ bounded $F_{t}$-adapted measurable setvalued $K_{C C}\left(R^{n}\right)$-process For any $(X(t))_{t \geq 0} \in L^{2}\left(\Omega, F_{t} ; K_{C C}\left(R^{n}\right)\right)$, we define

$$
\Gamma_{t}=\left\{\int_{0}^{t} x_{s} d w_{s}:\left(x_{s}\right)_{t \geq 0} \in S_{2}\left(X_{t}\right)\right\}
$$

for all $t \geq 0$, where $\left(w_{s}\right)_{t \geq 0}$ is a real valued Brownian motion with $w(0)=0$ a.s.

Lemma 3.2. (See [22) For any $(X(t))_{t \geq 0} \in L^{2}\left(\Omega, F_{t} ; K_{C C}\left(R^{n}\right)\right.$ ) and $t \geq 0$, there exists an $I_{t}(X) \in K_{C C}\left(R^{n}\right)$ such that $S^{1}\left(I_{t}(X)\right)=\overline{d e} \Gamma_{t}$

Definition 3.3. The set-valued stochastic process $\left(I_{t}(X)\right)_{t \geq 0}$ defined as above (see Lemma 3.2) is called a stochastic integral of $(X(t))_{t \geq 0} \in L^{2}\left(\Omega, F_{t} ; K_{C C}\left(R^{n}\right)\right)$ with respect to a real valued Brownian motion $\left(w_{t}\right)_{t \geq 0}$ For every $t$, we denote

$I_{t}(X)=\int_{0}^{t} X_{s} d w_{s}, \quad$ or $\quad I_{t}(X)(\omega)=\overline{d e} \Gamma_{t}(\omega)=\left\{\int_{0}^{t} x_{s} d w_{s}:\left(x_{t}\right)_{t \geq 0} \in S_{2}\left(X_{t}\right)\right\}$.

Definition 3.4. Let a set-valued stochastic process $(X(t))_{t \geq 0} \in L^{2}\left(\Omega, F_{t} ; K_{C C}\left(R^{n}\right)\right)$, for any $\omega \in \Omega$, we define

$L_{t}(X)=\int_{0}^{t} X_{s} d s$, or $L_{t}(X)(\omega)=\int_{0}^{t} X_{s} d s=\left\{\int_{0}^{t} x_{s} d s:\left(x_{t}\right)_{t \geq 0} \in S_{2}\left(X_{t}\right)\right\}$.

Where $\int_{0}^{t} x_{s} d s$ is the Lebesgue integral and $\int_{0}^{t} X_{s} d s$ is called the Aumann type Lebesgue integral of set-valued stochastic process $(X(t))_{t \geq 0}$ with respect to time $t$.

Lemma 3.3. (See $[19,22,26,28]$ ) Suppose that $(X(t))_{t \geq 0} \in L^{2}\left(\Omega, F_{t} ; K_{C C}\left(R^{n}\right)\right)$. Then there exists sequence $\left\{\left(x_{t}^{i}\right)_{t \geq 0}: i=1,2, \ldots\right\}$ of $S_{2}\left(X_{t}\right)$ such that for every $t \geq 0$,

$$
X_{t}=X(t, \omega)=c l\left\{\left(x_{t}^{i}\right)_{t \geq 0}: i=1,2, \ldots\right\} \text { and } I_{t}(X)(\omega)=c l\left\{\int_{0}^{t} x_{s}^{i}(\omega) d w_{s}(\omega): i=1,2, \ldots\right\} \text { a.a. } \omega \in \Omega \text {. }
$$

Theorem 3.2. Suppose that $(X(t))_{t \geq 0} \in L^{2}\left(\Omega, F_{t} ; K_{C C}\left(R^{n}\right)\right)$. Then there exists sequence $\left\{\left(x_{i}^{i}\right)_{t \geq 0}: i=1,2, \ldots\right\}$ of $S_{2}\left(X_{t}\right)$ such that for every $t \geq 0$,

$$
X_{t}=X(t, \omega)=c l\left\{\left(x_{t}^{i}\right)_{t \geq 0}: i=1,2, \ldots\right\} \text { and } L_{t}(X)(\omega)=c l\left\{\int_{0}^{t} x_{s}^{i}(\omega) d s(\omega): i=1,2, \ldots\right\} \text { a.a. } \omega \in \Omega \text {. }
$$

Theorem 3.3. Let $(X(t))_{t \geq 0} \in L^{2}\left(\Omega, F_{t} ; K_{C C}\left(R^{n}\right)\right)$ Then for every $t \geq 0$, we have

$$
I_{t}(X) \in L^{2}\left(\Omega, F_{t} ; K_{C C}\left(R^{n}\right)\right)
$$

Since all selections of $I_{t}(X)$ are $F_{t^{-}}$measurable, $I_{t}(X)$ is $F_{t^{-}}$measurable. In this theorem, we have to prove $I_{t}(X)(\omega)$ are convex set and $L^{2-}$ bounded. Let $y, z \in S^{1}\left(I_{t}(X)\right)$. Then there two $F_{t^{-}}$ measurable partitions $A_{1}, A_{2}, \ldots, A_{n}, B_{1}, B_{2}, \ldots, B_{n}$ of $\Omega$ and $\left\{y^{i}: i=1,2, \ldots\right\},\left\{z^{i}: i=1,2, \ldots\right\}$ 
$\subset \Gamma_{t} \equiv\left\{\int_{0}^{t} x_{s} d w_{s}:\left(x_{t}\right)_{t \geq 0} \in S_{2}\left(X_{t}\right)\right\}$ such that $\left|y-\sum_{i=1}^{n} 1_{A_{i}} y^{i}\right|<\varepsilon$ and $\left|z-\sum_{i=1}^{n} 1_{B_{i}} z^{i}\right|<\varepsilon$, for any $0 \leq \alpha \leq 1$, we have

$$
\left|\alpha y+(1-\alpha) z-\alpha \sum_{i=1}^{n} 1_{A_{i}} y^{i}-(1-\alpha) \sum_{i=1}^{n} 1_{B_{i}} z^{i}\right| \leq \alpha \varepsilon+(1-\alpha) \varepsilon=\text { on }
$$

and $\alpha \sum_{i=1}^{n} 1_{A_{i}} y^{i}+(1-\alpha) \sum_{j=1}^{m} 1_{B_{j}} z^{j}=\sum_{(i, j)=(1,1)}^{(n, m)} 1_{D_{(i, j)}}\left\{\alpha y^{i}+(1-\alpha) z^{j}\right\}$. Where $D_{(i, j)}=A_{i} \cap B_{j}$. Since $\left\{D_{(i, j)}: i=1,2, \ldots, n, j=1,2, \ldots, m\right\} \quad$ is $\quad$ an $\quad F_{t}$-measurable partition $\quad$ of $\Omega \quad$ and $\left\{\alpha y^{i}+(1-\alpha) z^{j}\right\} \subset \Gamma_{t},\{\alpha y+(1-\alpha) z\} \in \overline{d e} \Gamma_{t}=S^{1}\left(I_{t}(X)\right)$. Hence $S^{1}\left(I_{t}(X)\right)$ is convex.Next we prove that for every $t \geq 0$, the random set $I_{t}(X)$ is $L^{2}$-bounded. Indeed, we use Lemma 3.3 there exists a sequence $\left\{\left(x_{t}^{i}\right)_{t \geq 0}: i=1,2, \ldots\right\}$ of $S_{2}\left(X_{t}\right)$ such that for every $t \geq 0$, $X_{t}=X(t, \omega)=c l\left\{\left(x_{t}^{i}\right)_{t \geq 0}: i=1,2, \ldots\right\}$ and $I_{t}(X)(\omega)=c l\left\{\int_{0}^{t} x_{s}^{i}(\omega) d w_{s}(\omega): i=1,2, \ldots\right\}$ a.a. $\omega \in \Omega$.

We have

$$
\begin{aligned}
& E\left[d_{H}^{2}\left(I_{t}(X), \theta^{n}\right)\right]=\int_{\Omega} d_{H}^{2}\left(I_{t}(X), \theta^{n}\right) d P(\omega)=\int_{\Omega} \sup _{i}\left\|\int_{0}^{t} x_{s}^{i}(\omega) d w_{s}(\omega)\right\|^{-} d P(\omega) \\
& =\sup _{\left(y_{i}\right)_{\Omega_{2}} \in S_{2}\left(X_{t}\right)} E\left[\left|\int_{0}^{t} y_{s} d w_{s}\right|^{2}\right] \leq 2 E\left[\int_{0}^{t} \sup _{\bar{y} \in X_{x}}\left\|\bar{y}_{s}\right\|^{2} d s\right] \\
& =2 E\left[\int_{0}^{t} d_{H}^{2}\left(X_{s}, \theta^{n}\right) d s\right] .
\end{aligned}
$$

By the fact that $(X(t))_{t 20} \in L^{2}\left(\Omega, F_{t} ; K_{C C}\left(R^{n}\right)\right)$ and Lemma 3.1, the right side is finite. Hence, the proof is completed.

Theorem 3.4. ([22]) Assume that $\left(X_{t} ; x_{0}, c\right)$ satisfies condition (C). Then $\left(I_{t}\left(X ; x_{0}, c\right)\right)_{t \geq 0}$ is a setvalued $\mathrm{F}_{\mathrm{t}}$-martingale

Below we formulate some properties of the set-valued stochastic integral defined above. we get the following results.

Definition 3.5. Let $X, Y \in L^{2}\left(\Omega, F, F_{t}, P ; K_{C C}\left(R^{n}\right)\right)$, we have the following definitions :

i) For every $(t, \omega) \in[0, \infty) \times \Omega, \Delta_{2}^{2}\left(X_{t}, \theta^{n}\right)=\int_{\Omega} d_{H}^{2}\left(X(t, \omega), \theta^{n}\right) d P=E\left[d_{H}^{2}\left(X_{t}, \theta^{n}\right)\right]$

ii) For every $(t, \omega) \in[0, \infty) \times \Omega, \Delta_{2}^{2}\left(X_{t}, Y_{t}\right)=\int_{\Omega} d_{H}^{2}(X(t, \omega), Y(t, \omega)) d P=E\left[d_{H}^{2}\left(X_{t}, Y_{t}\right)\right]$

Using the properties of the Hausdorff distance one can formulate the following results

\section{Lemma 3.4.}

i) If $X, Y, Z \in L^{2}\left(\Omega, F, F_{t}, P ; K_{C C}\left(R^{n}\right)\right)$, then $\Delta_{2}^{2}(X+Z, Y+Z) \leq \Delta_{2}(X, Y)$.

ii) If $X_{1}, X_{2}, \ldots, X_{n}, Y_{1}, Y_{2}, \ldots, Y_{n} \in L^{2}\left(\Omega, F, F_{t} ; K_{C C}\left(R^{n}\right)\right), n \in N$, then

$$
\Delta_{2}^{2}\left(X_{1}+X_{2}+\ldots+X_{n}, Y_{1}+Y_{2}+\ldots+Y_{n}\right) \leq n \sum_{k=1}^{n} \Delta_{2}^{2}\left(X_{k}, Y_{k}\right) .
$$


iii) If $X, Y \in L^{2}\left(\Omega, F, F_{t} ; K_{C C}\left(R^{n}\right)\right)$ and $l, k$ are constants, then

$$
\Delta_{2}^{2}(l X, k Y) \leq|l-k| \Delta_{2}^{2}(X, Y) .
$$

Theorem 3.5. The space $L^{2}\left(\Omega, F, F_{t} ; K_{C C}\left(R^{n}\right)\right)$ is a complete metric space with respect to the metric $\Delta_{2}$.

It is clearly from Definition 3.5 and $\left(K_{C C}\left(R^{n}\right), d_{H}\right)$ is a complete metric space, with $K_{C C}\left(R^{n}\right)$ is a complete and separable with respect to $d_{H}$.

\section{Theorem 3.6.}

Let $\left(X_{t}\right)_{t \geq 0^{\circ}}\left(Y_{t}\right)_{t z 0} \in L^{2}\left(\Omega, F_{t} ; K_{C C}\left(R^{n}\right)\right)$. Then it holds

$$
E\left[d_{H}^{2}\left(\int_{0}^{t} X_{s} d w_{s}, \int_{0}^{t} Y_{s} d w_{s}\right)\right] \leq 2 E\left[\int_{0}^{t} d_{H}^{2}\left(X_{s}, Y_{s}\right) d s\right]
$$

By Lemma 3.3, there exist countable sequences $\left\{\left(x_{i}^{i}\right)_{t \geq 0}: i=1,2, \ldots\right\}$ and $\left\{\left(y_{t}^{j}\right)_{t \geq 0}: j=1,2, \ldots\right\}$ in $S_{2}\left(X_{t}\right)$ and $S_{2}\left(Y_{t}\right)$, respecttively such that for any $t \geq 0$,

$$
X_{t}=X(t, \omega)=c l\left\{x_{t}^{i}=x^{i}(t, \omega): i=1,2, \ldots\right\}, Y_{t}=Y(t, \omega)=c l\left\{y_{t}^{j}=y^{j}(t, \omega): j=1,2, \ldots\right\}
$$

and

$$
I_{t}(X)(\omega)=\int_{0}^{t} X_{s} d w_{s}=c l\left\{\int_{0}^{t} x_{s}^{i} d w_{s}: i=1,2, \ldots\right\}
$$

$$
\begin{aligned}
& I_{t}(Y)(\omega)=\int_{0}^{t} Y_{s} d w_{s}=c l\left\{\int_{0}^{t} y_{s}^{i} d w_{s}: i=1,2, \ldots\right\} \quad \text { a.a. } \quad \omega \in \Omega . \quad \text { Using basic property } \\
& \max (u, v)=\frac{1}{2}(u+v)+\frac{1}{2}|u-v|, \text { we have }
\end{aligned}
$$

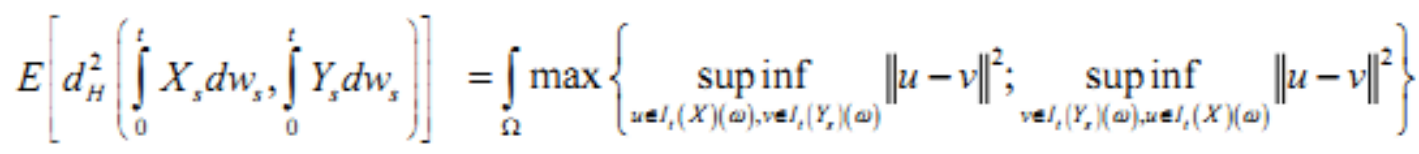

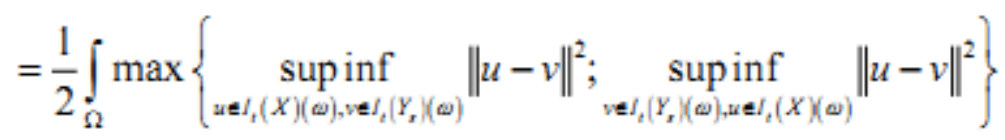

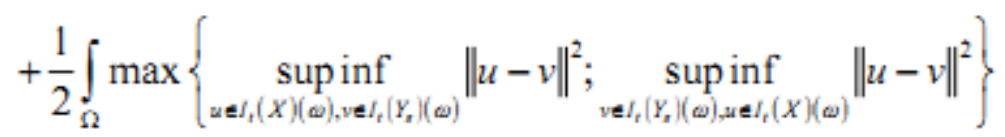

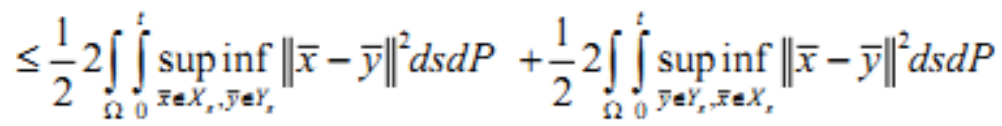

$$
\begin{aligned}
& +\frac{1}{2} 2 \int_{\Omega} \int_{0}^{t}\left|\operatorname{supinf}_{\bar{z} \in X_{x}, \bar{y} \in Y_{x}}\|\bar{x}-\bar{y}\|^{2}-\operatorname{supinf}_{\bar{y} \in Y_{x}, \bar{z} \in X_{x}}\|\bar{x}-\bar{y}\|^{2}\right| d s d P \\
& =2 \iint_{\Omega}^{t} \max \left\{\operatorname{supinf}_{\overline{\bar{x}} \in X_{x}, \bar{y} \in Y_{x}}\|\bar{x}-\bar{y}\|^{2}, \operatorname{supinf}_{\bar{y} \in Y_{n}, \bar{x} \in X_{x}}\|\bar{x}-\bar{y}\|^{2}\right\} d s d P \\
& =2 E\left[\int_{0}^{t} d_{H}^{2}\left(X_{s}, Y_{s}\right) d s\right] \text {. }
\end{aligned}
$$

The proof is completed. 
Corollary 3.5. Let $\left(X_{t}\right)_{t z 0} \in L^{2}\left(\Omega, F_{t} ; K_{C C}\left(R^{n}\right)\right)$. Then it holds

$$
E\left[d_{H}^{2}\left(\int_{0}^{t} X_{s} d w_{s}, \theta^{n}\right)\right] \leq 2 E\left[\int_{0}^{t} d_{H}^{2}\left(X_{s}, \theta^{n}\right) d s\right]
$$

Theorem 3.7. Let $\left(X_{t}\right)_{t \geq 0},\left(Y_{t}\right)_{t \geq 0} \in L^{2}\left(\Omega, F_{t} ; K_{C C}\left(R^{n}\right)\right)$. Then it holds

$$
E\left[d_{H}^{2}\left(\int_{0}^{t} X_{s} d s, \int_{0}^{t} Y_{s} d s\right)\right] \leq t E\left[\int_{0}^{t} d_{H}^{2}\left(X_{s}, Y_{s}\right) d s\right]
$$

By Theorem 3.2, there exist countable sequences $\left\{\left(x_{t}^{i}\right)_{t \geq 0}: i=1,2, \ldots\right\}$ and $\left\{\left(y_{t}^{j}\right)_{t \geq 0}: j=1,2, \ldots\right\}$ in $S_{2}\left(X_{t}\right)$ and $S_{2}\left(Y_{t}\right)$, respectively, such that for any $t \geq 0$,

$$
X_{t}=X(t, \omega)=c l\left\{x_{t}^{i}=x^{i}(t, \omega): i=1,2, \ldots\right\}, Y_{t}=Y(t, \omega)=c l\left\{y_{t}^{j}=y^{j}(t, \omega): j=1,2, \ldots\right\}
$$

and $L_{t}(X)(\omega)=\int_{0}^{t} X(s, \omega) d s=c l\left\{\int_{0}^{t} x^{i}(s, \omega) d s \mid i=1,2, \ldots\right\}$

and $L_{t}(Y)(\omega)=\int_{0}^{t} Y(s, \omega) d s=c l\left\{\int_{0}^{t} y^{j}(s, \omega) d s \mid j=1,2, \ldots\right\}$

Thus, we have

$$
\begin{aligned}
& E\left[d_{H}^{2}\left(\int_{0}^{t} X_{s} d s, \int_{0}^{t} Y_{s} d s\right)\right]=E\left[d_{H}^{2}\left(L_{t}(X)(\omega), L_{t}(Y)(\omega)\right)\right]
\end{aligned}
$$

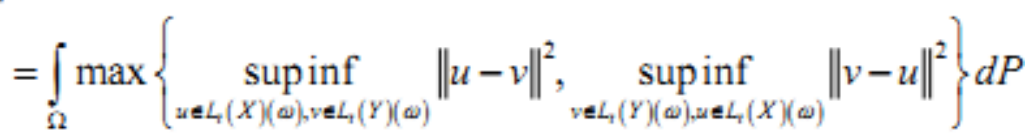

Noticing that,

$$
\operatorname{supinf}_{u \in L_{i}(X)(\omega), v \in L_{r}(Y)(\omega)}\|u-v\|^{2}=\operatorname{supinf}_{i \geq 1, v \in L_{r}(Y)(\omega)}\left\|\int_{0}^{t} x^{i}(s, \omega) d s-v\right\|^{2}
$$

Next we have

$$
\begin{aligned}
\inf _{j \geq 1}\left\|\int_{0}^{t}\left(x^{i}(s, \omega)-y^{j}(s, \omega)\right) d s\right\| & \leq \sqrt[2]{t^{2}} \inf _{j \geq 1} \int_{0}^{t}\left\|x^{i}(s, \omega)-y^{j}(s, \omega)\right\|^{2} d s \\
\leq t \int_{0}^{t} \operatorname{supinf}_{i \geq 1, j \geq 1}\left\|x^{i}(s, \omega)-y^{j}(s, \omega)\right\|^{2} d s & \\
& \leq t \int_{0}^{t} d_{H}^{2}(X, Y) d s
\end{aligned}
$$

Similarly, we obtain $\operatorname{supinf}_{v \in L_{,}(Y)(\omega), u \in L_{,}(X)(\omega)}\|v-u\|^{2} \leq t \int_{0}^{t} d_{H}^{2}(X, Y) d s$. Hence the proof is completed.

\section{Corollary 3.6.}

Let $\left(X_{t}\right)_{t \geq 0} \in L^{2}\left(\Omega, F_{t} ; K_{C C}\left(R^{n}\right)\right)$. Then it holds

$$
E\left[d_{H}^{2}\left(\int_{0}^{t} X_{s} d s, \theta^{n}\right)\right] \leq t E\left[\int_{0}^{t} d_{H}^{2}\left(X_{s}, \theta^{n}\right) d s\right]
$$

In next section, we present the existence, uniqueness properties of local solution under difference two conditions. 


\section{EXISTENCE AND UNIQUENESS PROPERTIES OF SOLUTION TO SET-VALUED STOCHASTIC DIFFERENTIAL EQUATIONS}

In this section we shall consider a set-valued stochastic differential equation under Hukuhara derivative (SSDE):

$$
X_{t}^{\prime}=F\left(t, X_{t}\right)+G\left(t, X_{t}\right) \xi(t), \quad X(0)=X_{0}
$$

where $X_{t}=X(t, \omega) \in K_{C C}\left(R^{n}\right), t \in[0, T] \subset R_{+}, \omega \in \Omega$ and $\xi(t)$ is one-dimensional "white noise",

with "white noise" is the time derivative of one-dimensional Wiener process, i.e., $\frac{d w_{t}}{d t}=\xi(t)$; $F:[0, T] \times \Omega \times K_{C C}\left(R^{n}\right) \rightarrow K_{C C}\left(R^{n}\right), F\left(t, X_{t}\right)=F(t, \omega, X(t, \omega))$ is measurable multifunction and Aumann integrably bounded.

$G:[0, T] \times \Omega \times K_{C C}\left(R^{n}\right) \rightarrow K_{C C}\left(R^{n}\right), G\left(t, X_{t}\right)=G(t, \omega, X(t, \omega))$ is measurable multifunction and Itô integrably bounded, $X_{0}: \Omega \rightarrow K_{C C}\left(R^{n}\right)$ is an $F_{0}$-measurable multifunction.

Definition 4.1. Let a set-valued stochastic process $X:[0, T] \times \Omega \rightarrow K_{C C}\left(R^{n}\right)$ satisfy:

i) $X_{t}=X(t, \omega) \in L^{2}\left(\Omega, F_{t}, P ; K_{c c}\left(R^{n}\right)\right)$

ii) for every $\omega \in \Omega, t \in[0, T] ; X:[0, T] \rightarrow L^{2}\left(\Omega, F_{t}, P ; K_{C C}\left(R^{n}\right)\right)$ is continuous mapping with respect to the metric $\Delta_{2}$;

iii) for every $t \in[0, T]$ :

$$
X_{t}=X_{0}+\int_{0}^{t} F\left(s, X_{s}\right) d s+\int_{0}^{t} G\left(s, X_{s}\right) d w_{s} \quad P \text {-a.e., }
$$

where $X_{0}: \Omega \rightarrow K_{C C}\left(R^{n}\right)$ is an $F_{0}$-measurable multifunction. Then $X_{t}$ is local solution of (4.1).

Definition 4.2. A solution $X_{t}$ to (4.1) is unique if for every $t \in[0, T]$ :

$$
\Delta_{2}^{2}\left(X_{t}, Y_{t}\right)=0 \quad \text { P.a.e., }
$$

where $Y_{t}$ is any solution to (4.1).

Lemma 4.1. (The Extension of Gronwall- Bellman Inequality)

Assume that the real functions $r(t)>0, a(t)>0, c(t)>0$ are continuous on $\left[t_{0}, T\right]$ satisfy:

$$
r(t) \leq a(t)+\int_{t_{0}}^{t} c(s) r(s) d s \text { then } r(t) \leq a(t)+\int_{t_{0}}^{t} a(s) c(s) \exp \left(\int_{s}^{t} c(l) d l\right) d s
$$

The following results we establish the existence and uniqueness of a local solution to SSDE (4.1) by using the Lipschitz condition (H2).

Next, we present the existence and uniqueness of a local solution to SSDE (4.1) by using the Lipschitz condition holds on bounded sets ( condition (H3)).

Assume that $F, G:[0, T] \subset R^{+} \times K_{C C}\left(R^{n}\right) \rightarrow K_{C C}\left(R^{n}\right)$, satisfy the following hypotheses:

(H1) for every set $A \in K_{C C}\left(R^{n}\right)$ the mappings

$$
F(\cdot, A), G(\cdot, A):[0, T] \times \Omega \rightarrow K_{C C}\left(R^{n}\right) \text { are nonanticipating multifunctions. }
$$

(H2) there exists constant $L>0$, such that

$$
\max \left\{d_{H}(F(t, A), F(t, \bar{A})), d_{H}(G(t, A), G(t, \bar{A}))\right\} \leq L \cdot d_{H}(A, \bar{A}) .
$$


(H3) there exists $\tau \in[0, T]$ for which the following condition hold:

a). for every $r>0$ there exists a constant $L_{r, t}>0$ such that $\max \left\{d_{H}(F(t, A), F(t, \bar{A})), d_{H}(G(t, A), G(t, \bar{A}))\right\} \leq L_{r, \tau} d_{H}(A, \bar{A})$, for all $t \in[0, \tau]$,

$A, \bar{A} \in B_{r}$, where $B_{r}=\left\{A \in K_{C C}\left(R^{n}\right) \mid d_{H}\left(A, \theta^{n}\right) \leq r\right\}$;

b).there exists $\tau \in[0, T]$ for which the following condition hold: there exists a constant

$S_{\tau}>0$ such that $\max \left\{d_{H}\left(F\left(t, \theta^{n}\right), \theta^{n}\right), d_{H}\left(G\left(t, \theta^{n}\right), \theta^{n}\right)\right\} \leq S_{\tau}$, for all $t \in[0, \tau]$.

Theorem 4.1. Assume that F, G satisfy (H1)-(H2), and

$$
\max \left\{\Delta_{2}^{2}\left(\int_{0}^{t} F\left(s, X_{0}\right) d s, \theta^{n}\right), \Delta_{2}^{2}\left(\int_{0}^{t} G\left(s, X_{0}\right) d w_{s}, \theta^{n}\right)\right\} \leq M \Delta_{2}^{2}\left(X_{0}, \theta^{n}\right),
$$

where $M>0$ is a constant. Then SSDE (4.1) has a unique local solution.

Proof. a/ Problem of existence is proved by integral expression (4.2). Let us define a sequence $X^{m}: \Omega \times[0, T] \rightarrow K_{C C}\left(R^{n}\right), m=0,1,2, \ldots \ldots$ of successive approximations as follows :

$X\left(t_{0}, \omega\right)=X_{0}$ and for $m=0,1,2, \ldots .$. and $X_{t}^{m}=X_{0}+\int_{0}^{t} F\left(s, X_{s}^{m-1}\right) d s+\int_{0}^{t} G\left(s, X_{s}^{m-1}\right) d w_{s}$.

By using Lemma 3.4, Corollary 3.5, Theorem 3.6, Corollary 3.6, Theorem 3.7, and assumptions of this theorem, we have

implies that,

$$
X_{t}^{1}=X_{0}+\int_{0}^{t} F\left(s, X_{0}\right) d s+\int_{0}^{t} G\left(s, X_{0}\right) d w_{s}
$$

$$
\begin{aligned}
& \Delta_{2}^{2}\left(X_{t}^{1}, X_{0}\right)=\Delta_{2}^{2}\left(\int_{0}^{t} F\left(s, X_{0}\right) d s+\int_{0}^{t} G\left(s, X_{0}\right) d w_{s}, \theta^{n}\right) \\
& \leq 2 \Delta_{2}^{2}\left(\int_{0}^{t} F\left(s, X_{0}\right) d s, \theta^{n}\right)+2 \Delta_{2}^{2}\left(\int_{0}^{t} G\left(s, X_{0}\right) d w_{s}, \theta^{n}\right)
\end{aligned}
$$

$$
\leq 4 M \Delta_{2}^{2}\left(X_{0}, \theta^{n}\right)<\infty
$$

Observe that for $m=1,2,3, \ldots .$. one has

$$
X_{t}^{m}=X_{0}+\int_{0}^{t} F\left(s, X_{x}^{m-1}\right) d s+\int_{0}^{t} G\left(s, X^{m-1}\right) d w_{s} ; X_{t}^{m+1}=X_{0}+\int_{0}^{t} F\left(s, X_{s}^{m}\right) d s+\int_{0}^{t} G\left(s, X_{x}^{m}\right) d w_{s}
$$

By using (H2), we get $\Delta_{2}^{2}\left(X_{t}^{m+1}, X_{t}^{m}\right) \leq 4(T+2) L^{2} \int_{0}^{t} \Delta_{2}^{2}\left(X_{x}^{m}, X_{x}^{m-1}\right) d s$ and consequently,

$$
\Delta_{2}^{2}\left(X_{t}^{m+1}, X_{t}^{m}\right) \leq\left(4(T+2) L^{2}\right)^{m-1} 4 M \Delta_{2}^{2}\left(X_{0}, \theta^{n}\right) \frac{t^{m-1}}{(m-1) !} \leq 4 M \Delta_{2}^{2}\left(X_{0}, \theta^{n}\right) \frac{\left(4(T+2) L^{2} T\right)^{m}}{m !}<\infty .
$$

Moreover for every $m$, the mapping $X^{m}(\cdot):[0, T] \rightarrow L^{2}\left(\Omega, F_{t}, P ; K_{C C}\left(R^{n}\right)\right)$ is continuous with respect to the metric $\Delta_{2}$. We claim that the sequence $\left\{X_{t}^{m}\right\}_{n=0}^{\infty}$ satisfies the Cauchy condition uniformly in $t$. Indeed, let us note that $\Delta_{2}^{2}\left(X_{t}^{l}, X_{t}^{k}\right) \leq\left(4 M \Delta_{2}^{2}\left(X_{0}, \theta^{n}\right)\right) \sum_{m=k+1}^{l} \frac{a^{m}}{m !}$, with $a=\left(4(T+2) L^{2} T\right)$. The series $\sum_{m=0}^{\infty} \frac{a^{m}}{m !}$ is convergent for every $a \in R_{+}$. Hence for any $\varepsilon>0$ there exists $n_{0} \in N$ such that for any $l, k>n_{0}$ it holds that $\sup _{t \in[0, T]} \Delta_{2}\left(X_{t}^{l}, X_{t}^{k}\right)<\varepsilon$. Thus $\left(X_{t}^{m}\right)$ 
atisfies a Cauchy condition uniformly in t. Now, we want to show that this limit process is solution to $\operatorname{SSDE}$ (4.1). Indeed, we show that $\left\{X_{t}^{m}\right\}$ satisfies the (4.2). We have

$\Delta_{2}^{2}\left(X_{t}, X_{0}+\int_{0}^{t} F\left(s, X_{s}\right) d s+\int_{0}^{t} G\left(s, X_{s}\right) d w_{s}\right) \leq 3 \Delta_{2}^{2}\left(X_{t}, X_{t}^{m}\right)$

$+3 \Delta_{2}^{2}\left(X_{t}^{m}, X_{0}+\int_{0}^{t} F\left(s, X_{s}^{m-1}\right) d s+\int_{0}^{t} G\left(s, X_{s}^{m-1}\right) d w_{s}\right)$

$+3 \Delta_{2}^{2}\left(\int_{0}^{t} F\left(s, X_{s}^{m-1}\right) d s+\int_{0}^{t} G\left(s, X_{s}^{m-1}\right) d w_{s}, \int_{0}^{t} F\left(s, X_{s}\right) d s+\int_{0}^{t} G\left(s, X_{s}\right) d w_{s}\right)$

We see $\Delta_{2}^{2}\left(X_{t}, X_{t}^{m}\right)$ uniformly converges to zero, and

$\Delta_{2}^{2}\left(X_{t}^{m}, X_{0}+\int_{0}^{t} F\left(s, X_{s}^{m-1}\right) d s+\int_{0}^{t} G\left(s, X_{s}^{m-1}\right) d w_{s}\right)$ is equal to zero. We consider

$\Delta_{2}^{2}\left(\int_{0}^{t} F\left(s, X_{s}^{m-1}\right) d s+\int_{0}^{t} G\left(s, X_{s}^{m-1}\right) d w_{s}, \int_{0}^{t} F\left(s, X_{s}\right) d s+\int_{0}^{t} G\left(s, X_{s}\right) d w_{s}\right)$

$\leq 2 \Delta_{2}^{2}\left(\int_{0}^{t} F\left(s, X_{s}^{m-1}\right) d s, \int_{0}^{t} F\left(s, X_{s}\right) d s\right)+2 \Delta_{2}^{2}\left(\int_{0}^{t} G\left(s, X_{s}^{m-1}\right) d w_{s}, \int_{0}^{t} G\left(s, X_{s}\right) d w_{s}\right)$

$\leq 4 L^{2}(T+2) \int_{0}^{t} \Delta_{2}^{2}\left(X_{s}^{m-1}, X_{s}\right) d s \leq 4 L^{2}(T+2) \sup _{t \in[0, T]} \Delta_{2}^{2}\left(X_{t}^{m-1}, X_{t}\right) \rightarrow 0$, as $m \rightarrow \infty$.

Therefore,

$$
\Delta_{2}^{2}\left(X_{t}, X_{0}+\int_{0}^{t} F\left(s, X_{s},\right) d s+\int_{0}^{t} G\left(s, X_{s}\right) d w_{s}\right)=0
$$

with any fixed $t \in[0, T]$. Hence the existence of solution is proved.

b/ If we have two stochastic set solutions $X_{t}, Y_{t}$ to $\operatorname{SSDE}(4.1)$ with condition $X_{0}=Y_{0}$. We have to prove that $\Delta_{2}^{2}\left(X_{t}, Y_{t}\right)=0$ P.a.e. We have:

$$
\begin{aligned}
& X_{t}=X_{0}+\int_{0}^{t} F\left(s, X_{s}\right) d s+\int_{0}^{t} G\left(s, X_{s}\right) d w_{s} \\
& Y_{t}=Y_{0}+\int_{0}^{t} F\left(s, Y_{s}\right) d s+\int_{0}^{t} G\left(s, Y_{s}\right) d w_{s}
\end{aligned}
$$

By using assumption (H2), we infer that

$$
\begin{gathered}
\Delta_{2}^{2}\left(X_{t}, Y_{t}\right) \leq 3 \Delta_{2}^{2}\left(X_{0}, Y_{0}\right)+ \\
3 \Delta_{2}^{2}\left(\int_{0}^{t} F\left(s, X_{s}\right) d s, \int_{0}^{t} F\left(s, Y_{s}\right) d s\right)+3 \Delta_{2}^{2}\left(\int_{0}^{t} G\left(s, X_{s}\right) d w_{s}, \int_{0}^{t} G\left(s, Y_{s}\right) d w_{s}\right) \\
\leq 3 \Delta_{2}^{2}\left(X_{0}, Y_{0}\right)+6(T+2) L^{2} \int_{0}^{t} \Delta_{2}^{2}\left(X_{s}, Y_{s}\right) d s
\end{gathered}
$$

Using Gronwall's inequality, we get

$$
\Delta_{2}^{2}\left(X_{t}, Y_{t}\right) \leq 3 \Delta_{2}^{2}\left(X_{0}, Y_{0}\right) \cdot \exp \left(6(T+2) L^{2} T\right)
$$

By $X_{0}=Y_{0}$, then $\Delta_{2}^{2}\left(X_{0}, Y_{0}\right) \equiv 0$. It implies $\Delta_{2}^{2}\left(X_{t}, Y_{t}\right)=0$ P.a.e.

The following theorem, we give a result that shows the existence and uniqueness of the local solution to SSDE (4.1). Specially, the Lipschitz condition used in this theorem is weaker than the global one in Theorem 4.1. The proof is similar to that in Theorem 4.1 but there are some differences. Hence, we only present brief. 
Theorem 4.2. Assume that $F, G$ satisfy condition (H1), (H3), and $X_{0} \in L^{2}\left(\Omega, F, P ; K_{C C}\left(R^{n}\right)\right)$ be an $F_{0}$-measurable multifunction. Then there exists a $T>0$ such that the $\operatorname{SSDE}(4.1)$ has a unique local solution on $[0, T]$.

Proof. By virtue of assumption (H3) there exists $\tau>0$ and there exists constant $L_{\tau, r}>0$ such that for every $(t, X) \in[0, \tau] \times B_{r}$ it holds

$\max \left\{d_{H}\left(F(t, X), \theta^{n}\right), d_{H}\left(G(t, X), \theta^{n}\right)\right\} \leq \max \left\{d_{H}\left(F(t, X), F\left(t, \theta^{n}\right)\right), d_{H}\left(G(t, X), G\left(t, \theta^{n}\right)\right)\right\}$

$+\max \left\{d_{H}\left(F\left(t, \theta^{n}\right), \theta^{n}\right), d_{H}\left(G\left(t, \theta^{n}\right), \theta^{n}\right)\right\} \leq r L_{\tau, r}+S_{\tau}$.

Proceeding similarly as in the proof of Theorem 4.1 , we define a sequence $X^{m}: \Omega \times[0, T] \rightarrow K_{C C}\left(R^{n}\right), m=0,1,2, \ldots .$. of successive approximations as follows :

$$
X\left(t_{0}, \omega\right)=X_{0} \text { and for } m=0,1,2, \ldots . . X^{m}=X_{0}+\int_{0}^{t} F\left(s, X_{s}^{m-1}\right) d s+\int_{0}^{t} G\left(s, X_{s}^{m-1}\right) d w_{s} .
$$

Next, let us define $T=\min \left\{\tau, \frac{r}{2 T(T+2) r L_{t, r}+S_{\tau}}\right\}$ By using assumptions of this theorem, we notice that the mappings $X^{m}$ are continuous and bounded for every $m \in 0,1,2 \ldots$ and we obtain estimate $\Delta_{2}^{2}\left(X_{t}^{m}, X_{t}^{m-1}\right) \leq\left(2 T(T+2) r L_{\tau, r}+S_{\tau}\right) L_{t, r}^{m-1} \frac{T^{m}}{m^{\prime}}$. Beside, we also get $t \rightarrow \Delta_{2}^{2}\left(X_{t}^{m}, X_{t}^{m-1}\right)$ is continuous. Thus, for same conclusions as in the proof Theorem 4.1, the sequence $\left\{X_{m}\right\}_{0}^{\infty}$ is uniformly convergent. Reasoning as in the proof of Theorem 4.1, we will claim that (4.2) be the solution of SSDE (4.1). Indeed, we have

$\Delta_{2}^{2}\left(X_{t}, X_{0}+\int_{0}^{t} F\left(s, X_{s}\right) d s+\int_{0}^{t} G\left(s, X_{s}\right) d w_{s}\right)=0$.

\section{SOME APPLICATIONS TO MODELS OF INTERVAL-VALUED STOCHASTIC DIFFERENTIAL EQUATIONS}

For example, in a finance market we consider some stock price at time $t$ denoted by $X_{t}$ which is a random variable defined on the probability space $(\Omega, F, P)$. Owing to the quick fluctuation of the stock price from time to time or to the existence of missing data, we may not precisely know the price $X_{t}(\omega)$ A possible model for this situation would be to give the upper and the lower prices (i.e. a margin for the error in the observation). Then we obtain an interval $X_{t}(\omega)=\left[X_{t}^{L}(\omega), X_{t}^{U}(\omega)\right]$, which is a special kind of a set-valued random variable, contains not only randomness but also impreciseness, and we assume $X_{t}(\omega)$ is certainly in this interval.

For example different, in environmental of the insurance premium, the risks is considered a main material of this industry. Beside that, the risks are random factors and associating with premiums, so insurance premiums should be built on the basis of risks to price insurance which could compensate and balance the damage occurs to their business costs. Otherwise, the risks are some kinds different and levels of influence are different, so they could influence to levels of price of the insurance premium. Hence, we may not precisely know the price of the insurance premium such that be beneficial to company of the insurance and customers. Then, in special the case we assume $X_{t}(\omega)$ is certainly in this interval which admissible prices

Example 5.1. (Stock prices) Let $X_{t}$ denote the price of a stock at time $t$, where $X_{t} \in K_{C C}(R)$ (i.e. interval-valued). We can model the evolution of $X_{t}$ in time by supposing that $X^{\prime}{ }_{t}$, the relative change of price, evolves according to the SSDE under the form

$$
X_{t}^{\prime}=\mu X_{t}+\delta X_{t} \xi(t), \quad X(0)=X_{0}=\left[X_{0}^{L}, X_{0}^{U}\right],
$$


for all $t \in[0, T]$, for certain constants $\mu, \delta>0$, called the drift and the volatility of the stock.

Since coefficients in (5.1) satisfy the conditions in Theorem 4.1, there is a unique solution $\left(X_{t}\right)_{t \geq 0}$ of (5.1). This means that for $t \in[0, T],\left(X_{t}\right)_{t \geq 0}$ satisfies the following interval-valued stochastic differential equation

$$
X_{t}^{\prime}=\mu\left[X_{t}^{L}, X_{t}^{U}\right]+\delta\left[X_{t}^{L}, X_{t}^{U}\right] \xi(t), \quad X(0)=X_{0}=\left[X_{0}^{L}, X_{0}^{U}\right],
$$

For all $t \in[0, T]$. That is,

$$
X_{t}=\left[X_{0}^{L}, X_{0}^{U}\right]+\int_{0}^{t} \mu\left[X_{s}^{L}, X_{s}^{U}\right]+\int_{0}^{t} \delta\left[X_{s}^{L}, X_{s}^{U}\right] d w_{s} .
$$

Since $\mu, \delta>0, X_{t}^{L}$ and $X_{t}^{U}$ are the solutions of the following stochastic differential system

$$
\begin{aligned}
& X_{t}^{L}=X_{0}^{L}+\int_{0}^{t} \mu X_{s}^{L}+\int_{0}^{t} \delta X_{s}^{L} d w_{s} . \\
& X_{t}^{U}=X_{0}^{U}+\int_{0}^{t} \mu X_{s}^{U}+\int_{0}^{t} \delta X_{s}^{U} d w_{s} .
\end{aligned}
$$

We can slove (5.4) and (5.5) by classic methods. Thus, the solutions of (5.4) and (5.5), respecttively are $\quad X_{t}^{L}=X_{0}^{L} \cdot \exp \left(\left(\mu-\frac{\delta^{2}}{2}\right) t+\delta W_{t}\right) \quad$ and $\quad X_{t}^{U}=X_{0}^{U} \cdot \exp \left(\left(\mu-\frac{\delta^{2}}{2}\right) t+\delta W_{t}\right)$. Its graphical representation can be seen in Figure 1.

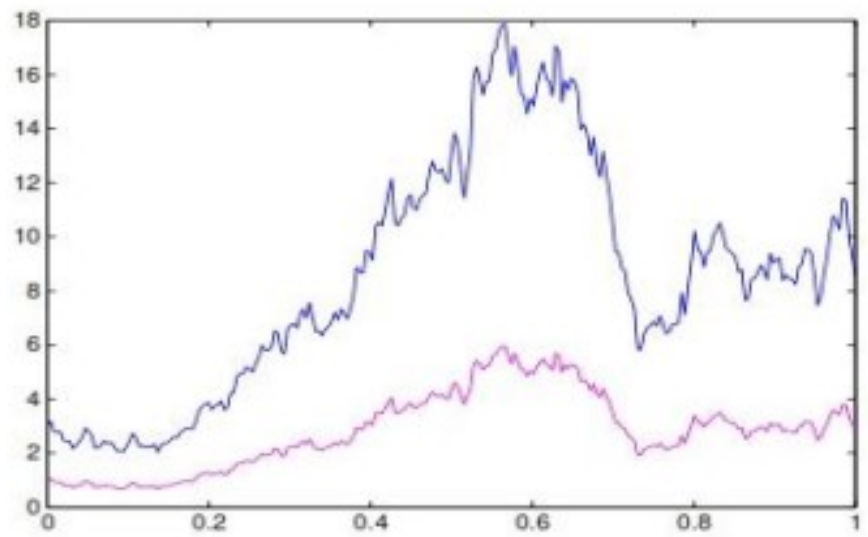

Solution of Example 5.1 in case $\mu=2, \delta=1$

Example 5.2. As another example, consider the Langevin equation

$$
X^{\prime}{ }_{t}=-\mu X_{t}+\delta \xi(t), \quad X(0)=X_{0}=\left[X_{0}^{L}, X_{0}^{U}\right],
$$

where $\mu$ and $\delta$ are positive constants.

We slove similar as in Example 5.1, then we have an interval-valued stochastic differential equation

$$
X_{t}^{\prime}=-\mu\left[X_{t}^{L}, X_{t}^{U}\right]+\delta \xi(t), \quad X(0)=X_{0}=\left[X_{0}^{L}, X_{0}^{U}\right]
$$

for all $t \in[0, T]$. That is, (7)

$$
X_{t}=\left[X_{0}^{L}, X_{0}^{U}\right]+\int_{0}^{t}-\mu\left[X_{s}^{L}, X_{s}^{U}\right]+\int_{0}^{t} \delta d w_{s} .
$$

Since $-[\alpha, \beta]=[-\beta,-\alpha]$ for all $\alpha \leq \beta \in R, X_{t}^{L}$ and $X_{t}^{U}$ are the solutions of the following real-valued stochastic differential system 


$$
\begin{aligned}
& X_{t}^{L}=X_{0}^{L}-\int_{0}^{t} \mu X_{s}^{U}+\int_{0}^{t} \delta d w_{s} . \\
& X_{t}^{U}=X_{0}^{U}-\int_{0}^{t} \mu X_{s}^{L}+\int_{0}^{t} \delta d w_{s} .
\end{aligned}
$$

Rewriting (5.6), (5.7) in the vector valued stochastic differential equation, we have

$$
X_{t}^{\prime}=\mu A X_{t}+B \xi(t), X(0)=X_{0} \text {. }
$$

where

$$
X_{t}=\left[\begin{array}{c}
X_{t}^{L} \\
X_{t}^{U}
\end{array}\right], A=\left[\begin{array}{cc}
0 & -1 \\
-1 & 0
\end{array}\right], B=\left[\begin{array}{l}
\delta \\
\delta
\end{array}\right], X_{0}=\left[\begin{array}{c}
X_{0}^{L} \\
X_{0}^{U}
\end{array}\right]
$$

The unique solution of stochastic differential equation (5.8) is given by,

$$
X_{t}=e^{\mu A t} X_{0}+\int_{0}^{t} e^{\mu(t-s) A} \delta d w_{s}=e^{\mu t}\left[\begin{array}{c}
X_{0}^{L}+\int_{0}^{t} e^{-\mu s} \delta d w_{s} \\
X_{0}^{U}+\int_{0}^{t} e^{-\mu s} \delta d d w_{s}
\end{array}\right]
$$

Hence $X_{t}=e^{\mu t}\left[X_{0}^{L}+\int_{0}^{t} e^{-\mu s} \delta d w_{s}, X_{0}^{U}+\int_{0}^{t} e^{-\mu s} \delta d w_{s}\right]$ is a unique solution of Example 5.2.

\section{ACKNOWLEDGMENTS}

The authors gratefully acknowledge the referees for their careful reading and many valuable remarks which improved the presentation of the paper. This work was supported by The National Foundation for Science and Technology Development Viet Nam (NAFOSTED).

\section{REFERENCES}

[1] F.S. De Blasi, F. Iervolino, Equazioni differenziali con soluzioni a valore compatto convesso, Journal of integral euqations and applications, Boll. Unione Mat. Ital. 4 (2) (1969), pp 194-501.

[2] M. Puri, D. Ralescu, Differentials of fuzzy functions, J. Math. Anal. Appl., 91 (1983), pp 552558.

[3] O. Kaleva, Fuzzy differential equations, J. Fuzzy Sets and Systems, 24 (1987), pp 301-317.

[4] Jong Yeoul Park and Jae Ug Jeong, On existence and uniqueness of solutions of fuzzy integrodifferential equations, Indian J. pure appl. Math, Vol. 34, Issue 10 (2003), pp 1503 -1512.

[5] T. Gnana Bhaskar, V. Lakshmikantham, Set differential equations and Flow invariance, J. Appl. Anal., 82 (2) (2003), pp 357-368.

[6] V. Lakshmikantham, T. Gnana Bhaskar and J. Vasundhara Devi, Theory of set differential equations in metric spaces, Cambridge Scientific Publisher, UK, 2006.

[7] V. Lakshmikantham, A.A. Tolstonogov, Existence and interrelation between set and fuzzy differential equations, J. Nonlinear Analysis (TMA), 55 (3) (2003), pp 255-268.

[8] L. Stefanini, L. Sorini, M. L. Guerra, Parametric representation of fuzzy numbers and application to fuzzy calculus, J. Fuzzy Sets and Systems, 157 (2006), pp 2423-2455.

[9] R.P. Agarwal, D. O'Regan, Existence for set differential equations via mul-tivalued operator equations, Differential equations and applications. Vol. 5, pp 1-5, Nova Sci. Publ., New York, (2007).

[10] F.S. de Blasi, V. Lakshmikantham, T. G. Bhaskar, An existence theorem for set differential inclusions in a semilinear metric space, Control Cybernet. 36 (3) (2007), 571-582. 
[11] L. Stefanini, A generalization of Hukuhara difference, in D. Dubois , M.A.Lubiano, H. Prade, M.A. Gil, P. Grzegorzewski, O. Hryniewicz (Eds), Soft Methods for Handling Variability and Imprecision, Series on Advances in Soft Computing(vol 48), Springer, 2008.

[12] Y. Chalco-Cano, H. Roman-Flores, On new solutions of fuzzy differential equations, J. Chaos, Solitons \& Fractals, 38(2008), pp 112-119.

[13] L. Stefanini, B. Bede, Generalized Hukuhara differentiability of interval-valued functions and interval differential equations, J. Nonlinear Analysis (TMA) 71 (2009), pp 1311-1328, doi:10.1016/j.na.2008.12.005.

[14] N.D.Phu, N.V.Hoa, H.Vu, On Comparisons of Set solutions for Fuzzy Control IntegroDifferential Systems, Journal of Advanced Research in Applied Mathematics (JARAM), (Accepted 22 December 2011) (Article in Press).

[15] N.V.Hoa., N.D.Phu, On maximal and minimal solutions for Set-valued Differential Equations with Feedback Control, Hindawi Publishing Corporation, J. Abstract and Applied Analysis

[16] J. Vasundhara Devi, Generalized monotone iterative technique for set differential equations involving causal operators with memory,Int J Adv Eng Sci Appl Math, doi 10.1007/s12572-0110031-1 (2011).

[17] T. Allahviranloo, A. Amirteimoori, M. Khezerloo, S. Khezerloo, A New Method for Solving Fuzzy Volterra Integro-Differential Equations, Australian Journal of Basic and Applied Sciences, Vol. 5, Issue 4 (2011), pp 154 -164.

[18] P.Protter, Stochastic Integration and Differential Equations: A New Approach, Springer Verlag, New York, 1990.

[19] F.Hiai, H.Umegaki, Integrals, conditional expectations and martingales for multivalued functions, J. Multivalued Anal. 7 (1977), pp 147 - 182.

[20] J.P.Aubin, G.Da Prato, The viability theorem for stochastic differential inclusion, Stoch. Anal. Appl. 15(5) (1997), pp 783- 800.

[21] J.Motyl, Stochastic functional inclusion driven by semimartingle, Stochastic Anal. Appl, 16 (3) (1998), pp $517-532$.

[22] E.J.Jung, J.H.Kim, On set-valued Stochastic integrals, Stoch. Anal. Appl. 21(2) (2003), pp 401-418.

[23] Tolstonogov A., Differential Inclusions in a Banach Space, Kluweer Acad. pul., Dordrecht, 2000.

[24] Mariusz Michta, J.Motyl, Differential selection of multifunctions and their applications, J. Nonlinear Analysis: 66(2) (2007), pp 536 - 545.

[25] J.Zhang, Set-valued stochastic integrals with respect to a real valued martingales,in: Soft Method for Handling Variability and Imprecision, Springer, Berlin 2008, pp 253 -259.

[26] J.Li, S.li Set-valued stochastic Lebesgue integral and representation theorems, Int.J.Comput.Intelligence Syst. 1 (2) (2008), pp 177 - 187.

[27] J.Zhang, S.Li, I.Mitoma, Y.Okazaki,On set-valued stochastic integrals in an M-type 2 Banach space, J. math.Anal.Appl, 350 (2009), pp 216 -233.

[28] Marek T. Malinowski, Mariusz Michta, Stochastic set differential equations, J. Nonlinear Analysis: TMA, 72 (2010), pp 1247 - 1256.

[29] Marek T. Malinowski, Mariusz Michta, Stochastic Fuzzy differential equations with an application, Kybernetika, Vol 47 (2011), pp 123 - 143.

[30] Mariusz Michta, On set-valued stochastic integrals and fuzzy stochastic equations, Fuzzy Set and Systems: (2011),doi:10.1016/j.fss.2011.01.007. 